\title{
A Highly Efficient Iridium Catalyst for Asymmetric Transfer Hydrogenation of Aromatic Ketones Under Base-free Conditions
}

Zhen-Rong Dong, Yan-Yun Li, Jian-Shan Chen, Bao-Zhu Li, Yan Xing, Jing-Xing Gao*

State Key Laboratory of Physical Chemistry of Solid Surfaces and Department of Chemistry, College of Chemistry and Chemical Engineering, Xiamen University, Xiamen 361005, P R China

cuihua@jingxian.xmu.edu.cn

\section{Supporting Information}

1. General

2. Synthesis and IR spectra of $\mathrm{Ru}, \mathrm{Rh}$, Ir hydride complexes

3. A typical procedure for asymmetric transfer hydrogenation with $\mathrm{S} / \mathrm{C}=$ 200

4. A typical procedure for asymmetric transfer hydrogenation with $\mathrm{S} / \mathrm{C}=$ 2000

5. GC analytical data for chiral aromatic alcohols

6. Reference

1. General

All experiments were carried out in a nitrogen atmosphere with Schlenk techniques, all solvents were dried and purified according to standard methods before use. All 
ketones were purchased from Aldrich, Kanto Chemical Co. (Japan) and Schuchardt (Germany), and used directly without further purification. Chiral ligand S-Binam was purchased from Aldrich. IR spectra were recorded on a Nicolet AVATAR FT-IR360 spectrometer using $\mathrm{KBr}$ pellets. Enantiomeric ratio was determined by chiral GC on Chrompack CP-cyclodextrin- $\beta-236-M-19$ column, Chiraldex G-TA column or CP-Chiralsil-Dex CB column.

2. Synthesis and IR spectra of Ru, Rh, Ir hydride complexes

$\operatorname{RuHCl}(\mathrm{CO})\left(\mathrm{PPh}_{3}\right)_{3}, \mathrm{RhHCO}\left(\mathrm{PPh}_{3}\right)_{3}, \mathrm{IrH}_{3}\left(\mathrm{PPh}_{3}\right)_{3}$ and $\operatorname{IrHCO}\left(\mathrm{PPh}_{3}\right)_{3}$ were synthesized according to literature ${ }^{1}$. RuHCl $(\mathrm{CO})\left(\mathrm{PPh}_{3}\right)_{3}$ was obtained as white microcrystals, $v_{\mathrm{co}}, v_{\mathrm{RuH}} 2015 \mathrm{~cm}^{-1}$, $1930 \mathrm{~cm}^{-1}$, $1888 \mathrm{~cm}^{-1}$ (lit., $2010 \mathrm{~cm}^{-1}, 1920 \mathrm{~cm}^{-1}, 1900 \mathrm{~cm}^{-1}$ ); RhHCO $\left(\mathrm{PPh}_{3}\right)_{3}$ was obtained as yellow microcrystals, $v_{\mathrm{CO}} 1921 \mathrm{~cm}^{-1}, v_{\mathrm{RhH}} 2037 \mathrm{~cm}^{-1}$ (lit. , ${ }^{2} v_{\mathrm{CO}} 1920 \mathrm{~cm}^{-1}, v_{\mathrm{RhH}} 2040 \mathrm{~cm}^{-1}$ ); mer- $\mathrm{IrH}_{3}\left(\mathrm{PPh}_{3}\right)_{3}$ was obtained as white microcrystals, $v_{\mathrm{IrH}} 1746$ and $2130 \mathrm{~cm}^{-1}\left(\mathrm{lit} .{ }^{1} v_{\mathrm{IrH}}\right.$ 1740 and $\left.2130 \mathrm{~cm}^{-1}\right)$; IrHCO $\left(\mathrm{PPh}_{3}\right)_{3}$ was obtained as yellow needles, $v_{\mathrm{CO}} 1918 \mathrm{~cm}^{-1}, v_{\text {IrH }} 2120$ $\mathrm{cm}^{-1}$ (lit. , ${ }^{1} v_{\mathrm{CO}} 1915 \mathrm{~cm}^{-1}, v_{\text {IrH }} 2120 \mathrm{~cm}^{-1}$ ).

3. A typical procedure for asymmetric transfer hydrogenation with $\mathrm{S} / \mathrm{C}=$ 200

A small-scale reaction with $\mathrm{S} / \mathrm{C}=200$ was conducted in a schlenk tube. An Example is given by asymmetric transfer hydrogenation of propiophenone using IrHCO $\left(\mathrm{PPh}_{3}\right)_{3}$ /ligand 2 as the catalyst.

The complex IrHCO $\left(\mathrm{PPh}_{3}\right)_{3}(2.5 \mathrm{mg}, 0.0025 \mathrm{mmol})$ and the chiral ligand $2(1.7 \mathrm{mg}, 0.0026$ mmol) were placed in a schlenk tube equipped with a Teflon-coated magnetic stirring bar, air present in the tube was removed by ten vacuum-filling with nitrogen cycles. Isopropanol $\left(5 \mathrm{~cm}^{3}\right)$ was then added into this tube, the mixture was stirred at $75^{\circ} \mathrm{C}$ for $30 \mathrm{~min}$. To this solution propiophenone $(67.1 \mathrm{mg}, 0.5 \mathrm{mmol})$ was added, the mixture was then stirred at this temperature for $0.5 \mathrm{~h}$. Samples were taken out of the reaction solution, passed through a column of silica and analyzed by GC.

4. A procedure for asymmetric transfer hydrogenation of propiophenone with $S / C=2000$

A large-scale reaction with $\mathrm{S} / \mathrm{C}=2000$ was conducted in a $150-\mathrm{mL}$ three-necked flask.

The complex IrHCO $\left(\mathrm{PPh}_{3}\right)_{3}(2.5 \mathrm{mg}, 0.0025 \mathrm{mmol})$ and the chiral ligand $2(1.7 \mathrm{mg}, 0.0026$ mmol) were placed in a $150-\mathrm{mL}$ three-necked flask equipped with a Teflon-coated magnetic stirring bar and a condenser, air present in the tube was removed by ten vacuum-filling with nitrogen cycles. Isopropanol $\left(5 \mathrm{~cm}^{3}\right)$ was then added into this flask, the mixture 
was stirred at $70^{\circ} \mathrm{C}$ for $30 \mathrm{~min}$. To this solution another isopropanol $\left(45 \mathrm{~cm}^{3}\right)$ and propiophenone $\left(671 \mathrm{mg}, 5 \mathrm{mmol}\right.$ ) was added, the mixture was then heated up to $82^{\circ} \mathrm{C}$ and stirred at this temperature for $2 \mathrm{~h}$. Samples were taken out of the reaction solution, passed through a column of silica and analyzed by GC.

5. GC analytical data for reduction of prochiral aromatic ketones (a)<smiles>CCC(=O)c1ccccc1</smiles>

GC analysis: Chrompack CP-cyclodextrin- $\beta-236-\mathrm{M}-19$ column, $0.25 \mathrm{~mm} \times 50 \mathrm{~m}$, , column temperature $=110^{\circ} \mathrm{C}$ (isothermal), inject temperature $=250^{\circ} \mathrm{C}$, detector temperature $=250{ }^{\circ} \mathrm{C}$, inlet pressure $=0.12 \mathrm{MPa} ; t_{s}=14.0 \mathrm{~min}, t_{1}=23.8 \mathrm{~min}, t_{2}=25.0 \mathrm{~min}$. ( $t_{s}$ : retention time of substrate; $t_{1}, t_{2}$ : retention time of chiral alcohol.);

GC trace of reduction of propiophenone catalyzed by $\mathrm{RhH}(\mathrm{CO})\left(\mathrm{PPh}_{3}\right)_{3} / 2 \mathrm{~b}$

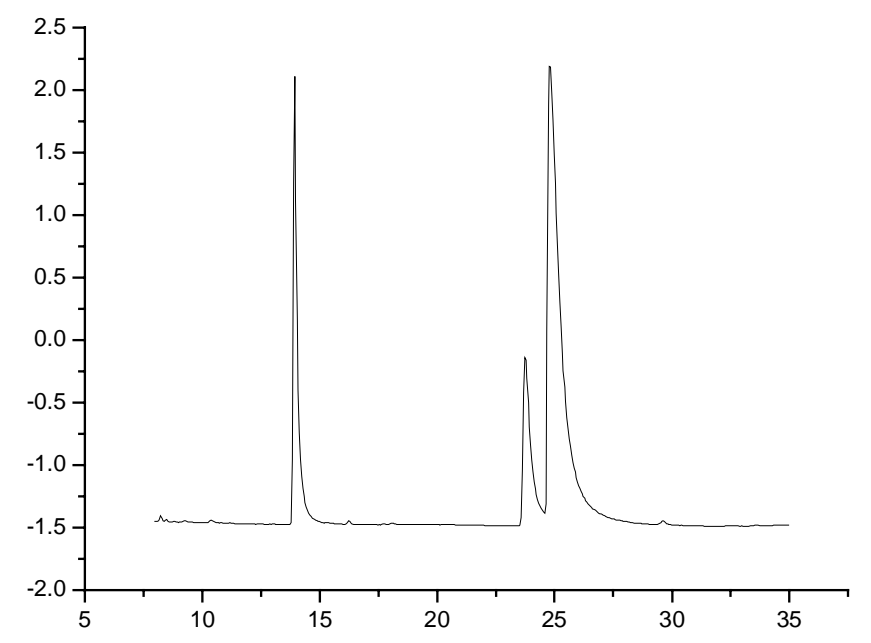

GC trace of reduction of propiophenone catalyzed by mer- $\mathrm{IrH}_{3}\left(\mathrm{PPh}_{3}\right)_{3} / 2 \mathrm{~b}$ 


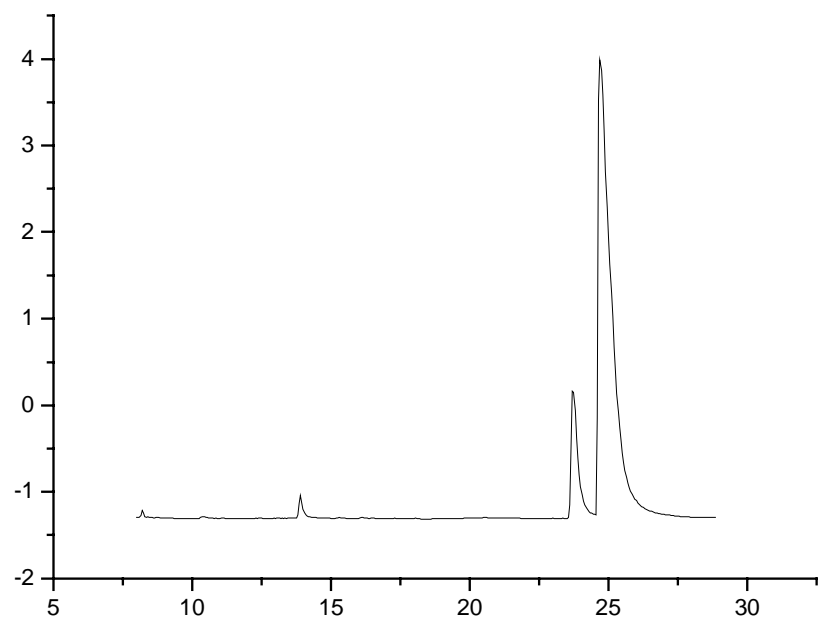

GC trace of reduction of propiophenone catalyzed by $\operatorname{IrH}(\mathrm{CO})\left(\mathrm{PPh}_{3}\right)_{3} / 2 \mathrm{~b}$

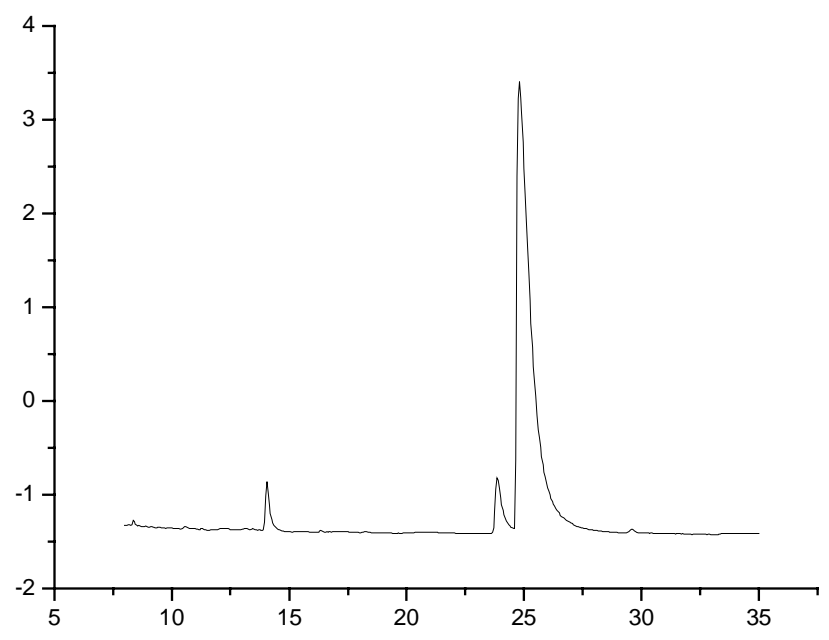

GC trace of large-scale $(\mathrm{S} / \mathrm{C}=2000)$ reduction of propiophenone catalyzed by 


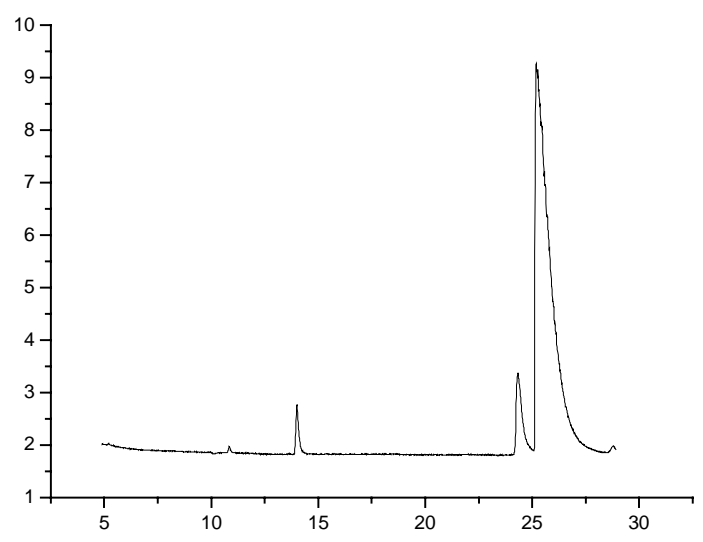

GC trace of reduction of propiophenone catalyzed by $\operatorname{IrH}(\mathrm{CO})\left(\mathrm{PPh}_{3}\right)_{3} / 1 \mathrm{~b}$

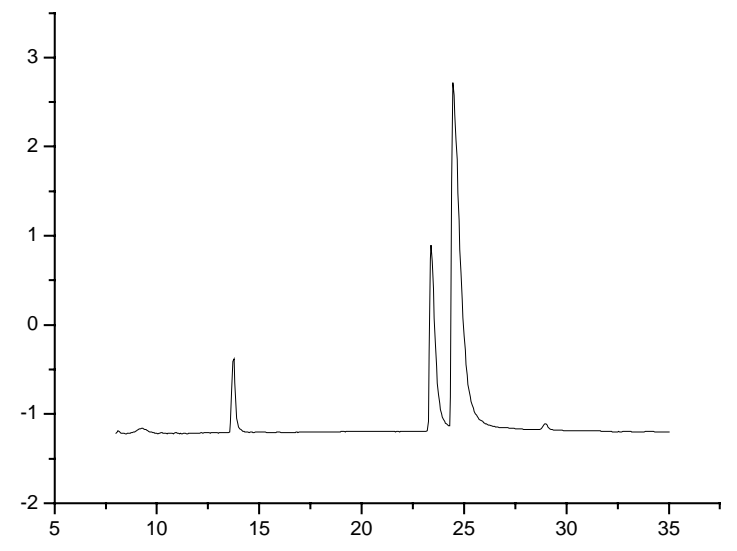

GC trace of reduction of propiophenone catalyzed by $\operatorname{IrH}(\mathrm{CO})\left(\mathrm{PPh}_{3}\right)_{3} / 4 \mathrm{~b}$

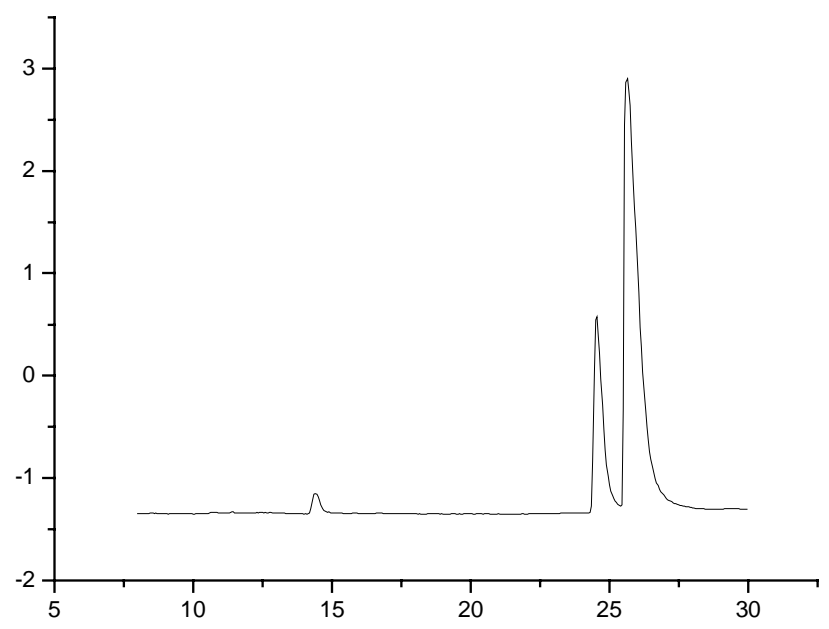

GC trace of reduction of propiophenone catalyzed by $\operatorname{IrH}(\mathrm{CO})\left(\mathrm{PPh}_{3}\right)_{3}$ without adding 


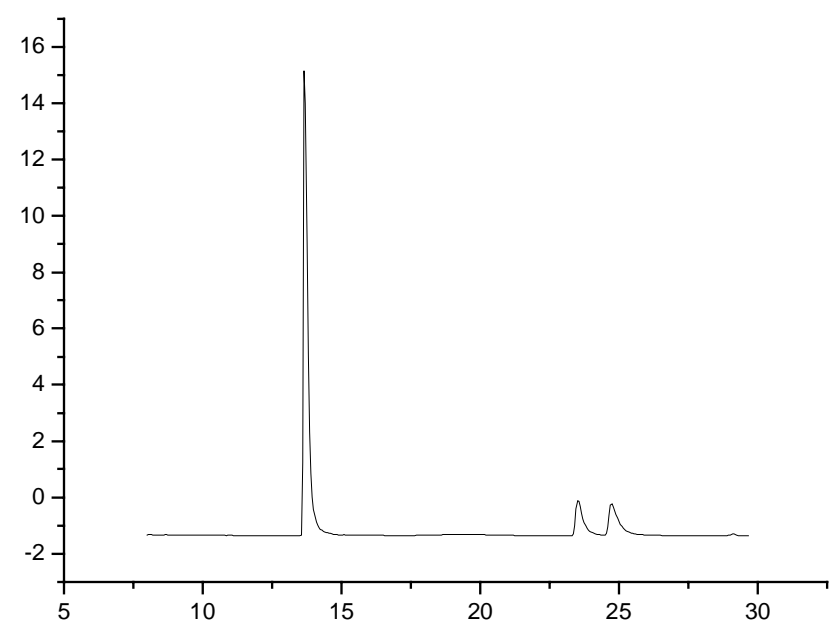

(b)<smiles>CC(C)C(=O)c1ccccc1</smiles>

GC analysis: Chrompack CP-cyclodextrin- $\beta-236-\mathrm{M}-19$ column, $0.25 \mathrm{~mm} \times 50 \mathrm{~m}$, column temperature $=115{ }^{\circ} \mathrm{C}$ (isothermal), inject temperature $=250^{\circ} \mathrm{C}$, detector temperature $=250^{\circ} \mathrm{C}$, inlet pressure $=0.08 \mathrm{MPa} ; t_{s}=13.2 \mathrm{~min}, t_{1}=27.5 \mathrm{~min}, t_{2}=27.9 \mathrm{~min}$. ( $t_{s}$ : retention time of substrate; $t_{1}, t_{2}$ : retention time of chiral alcohol.)

GC trace of reduction of ketone $6 \mathrm{~b}$ catalyzed by $\operatorname{IrH}(\mathrm{CO})\left(\mathrm{PPh}_{3}\right)_{3} / 2 \mathrm{~b}$

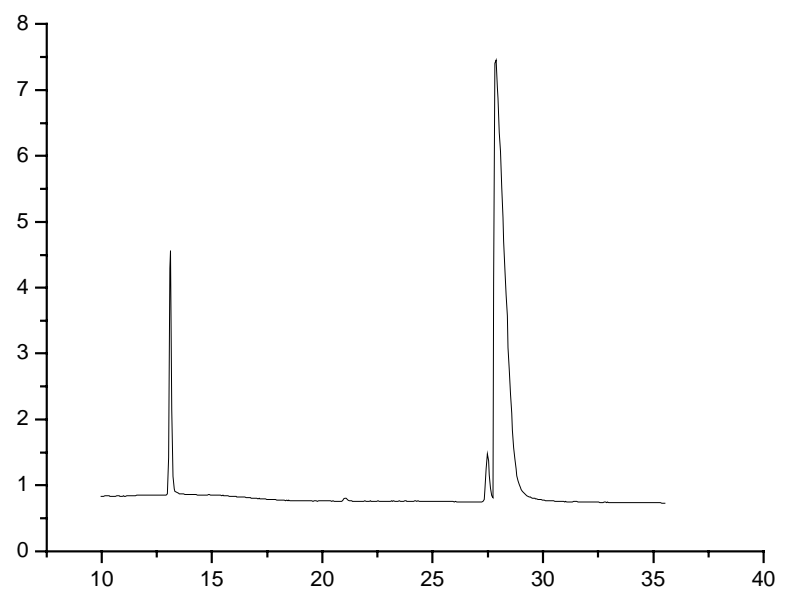

(c) 
<smiles>CC(C)(C)C(=O)c1ccccc1</smiles>

GC analysis: Chiraldex G-TA column, $0.25 \mathrm{~mm}$ x $30 \mathrm{~m}$, column temperature = $110{ }^{\circ} \mathrm{C}$ (isothermal), inject temperature $=250{ }^{\circ} \mathrm{C}$, detector temperature $=250{ }^{\circ} \mathrm{C}$, inlet pressure $=0.1 \mathrm{MPa} ; t_{s}=5.95 \mathrm{~min}, t_{1}=10.7 \mathrm{~min}, t_{2}=11.0 \mathrm{~min}$. (t s $_{\mathrm{s}}$ retention time of substrate; $t_{1}, t_{2}$ : retention time of chiral alcohol.)

$\mathrm{GC}$ trace of reduction of ketone $6 \mathrm{c}$ catalyzed by $\operatorname{IrH}(\mathrm{CO})\left(\mathrm{PPh}_{3}\right)_{3} / 2 \mathrm{a}$

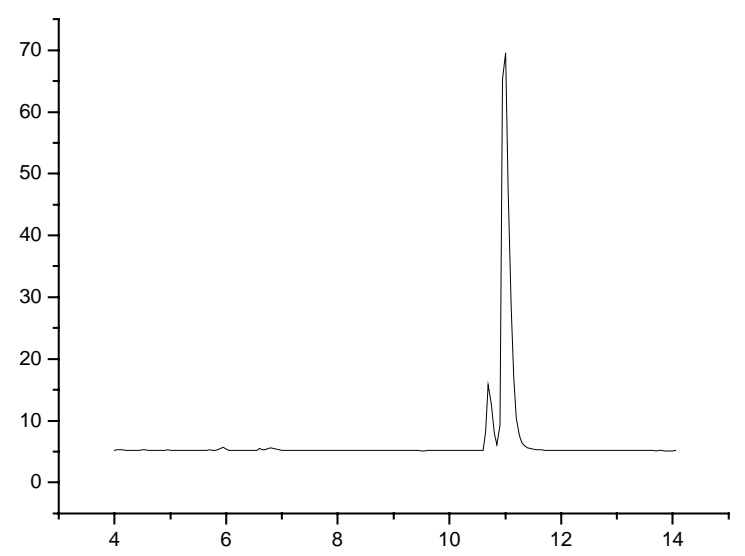

(d)<smiles>CC(=O)c1ccccc1[N+](=O)[O-]</smiles>

GC analysis: Chrompack CP-cyclodextrin- $\beta-236-\mathrm{M}-19$ column, $0.25 \mathrm{~mm}$ x $50 \mathrm{~m}$, column temperature $=150{ }^{\circ} \mathrm{C}$ (isothermal), inject temperature $=250{ }^{\circ} \mathrm{C}$, detector temperature $=250{ }^{\circ} \mathrm{C}$, inlet pressure $=0.12 \mathrm{MPa} ; t_{\mathrm{s}}=18.0 \mathrm{~min}, t_{1}=34.2 \mathrm{~min}, t_{2}=39.2 \mathrm{~min}$. $\left(t_{\mathrm{s}}\right.$ : retention time of substrate; $t_{1}, t_{2}$ : retention time of chiral alcohol.)

GC trace of reduction of ketone o-6d catalyzed by $\operatorname{IrH}(\mathrm{CO})\left(\mathrm{PPh}_{3}\right)_{3} / \mathbf{2 b}$

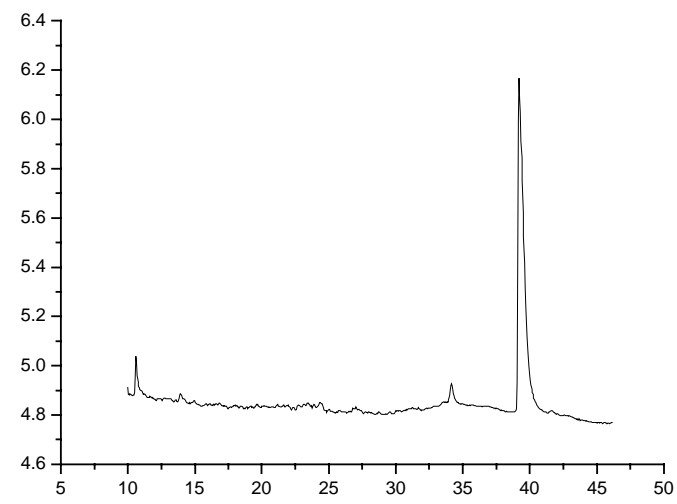

(e) 


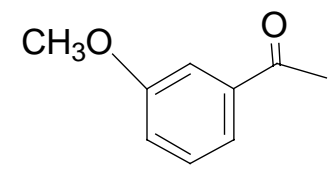

GC analysis: Chrompack CP-cyclodextrin- $\beta-236-\mathrm{M}-19$ column, $0.25 \mathrm{~mm} \times 50 \mathrm{~m}$, column temperature $=135{ }^{\circ} \mathrm{C}$ (isothermal), inject temperature $=250{ }^{\circ} \mathrm{C}$, detector temperature $=250{ }^{\circ} \mathrm{C}$, inlet pressure $=0.12 \mathrm{MPa} ; t_{\mathrm{s}}=14.0 \mathrm{~min}, t_{1}=21.8 \mathrm{~min}, t_{2}=22.8 \mathrm{~min}$. $\left(t_{s}:\right.$ retention time of substrate; $t_{1}, t_{2}$ : retention time of chiral alcohol.) GC trace of reduction of ketone m-6e catalyzed by $\operatorname{IrH}(\mathrm{CO})\left(\mathrm{PPh}_{3}\right)_{3} / \mathbf{2 b}$

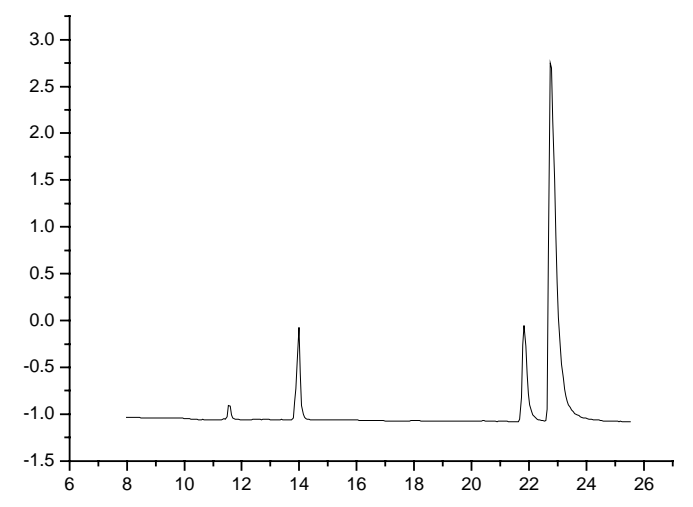

(f)<smiles>CC(=O)C(c1ccccc1)c1ccccc1</smiles>

GC analysis: CP-Chiralsil-Dex CB column, $0.25 \mathrm{~mm} \times 25 \mathrm{~m}$, column temperature $=150$ ${ }^{\circ} \mathrm{C}$ (isothermal), inject temperature $=250{ }^{\circ} \mathrm{C}$, detector temperature $=250{ }^{\circ} \mathrm{C}$, inlet pressure $=0.1 \mathrm{MPa} ; t_{s}=13.8 \mathrm{~min}, t_{1}=18.45 \mathrm{~min}, t_{2}=18.7 \mathrm{~min} .\left(t_{s}\right.$ : retention time of substrate; $t_{1}, t_{2}$ : retention time of chiral alcohol.)

GC trace of reduction of ketone 8 catalyzed by $\operatorname{IrH}(\mathrm{CO})\left(\mathrm{PPh}_{3}\right)_{3} / 2 \mathrm{a}$

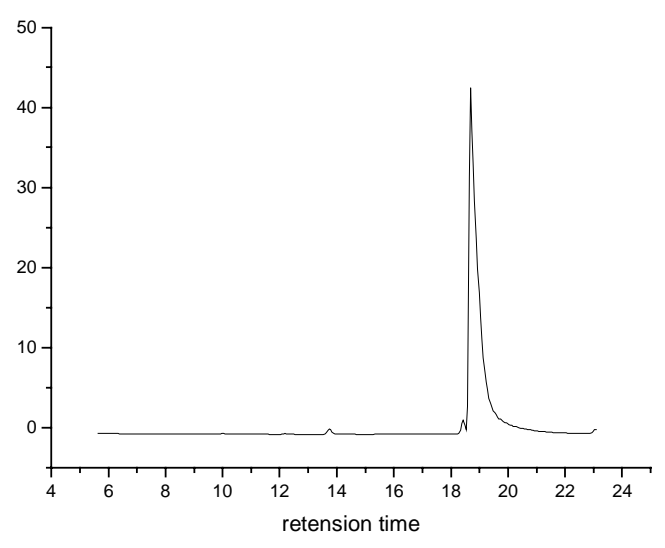


(i)

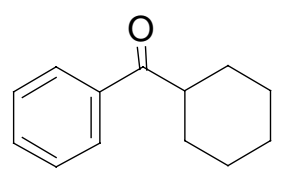

GC analysis: CP-Chiralsil-Dex CB column, $0.25 \mathrm{~mm} \times 25 \mathrm{~m}$, column temperature $=130$ ${ }^{\circ} \mathrm{C}$ (isothermal), inject temperature $=250{ }^{\circ} \mathrm{C}$, detector temperature $=250{ }^{\circ} \mathrm{C}$, inlet pressure $=0.1 \mathrm{MPa} ; t_{s}=20.3 \mathrm{~min}, t_{1}=44.8 \mathrm{~min}, t_{2}=48.1 \mathrm{~min} .\left(t_{s}\right.$ : retention time of substrate; $t_{1}, t_{2}$ : retention time of chiral alcohol.)

GC trace of reduction of ketone 9 catalyzed by $\operatorname{IrH}(\mathrm{CO})\left(\mathrm{PPh}_{3}\right)_{3} / 2 \mathrm{~b}$

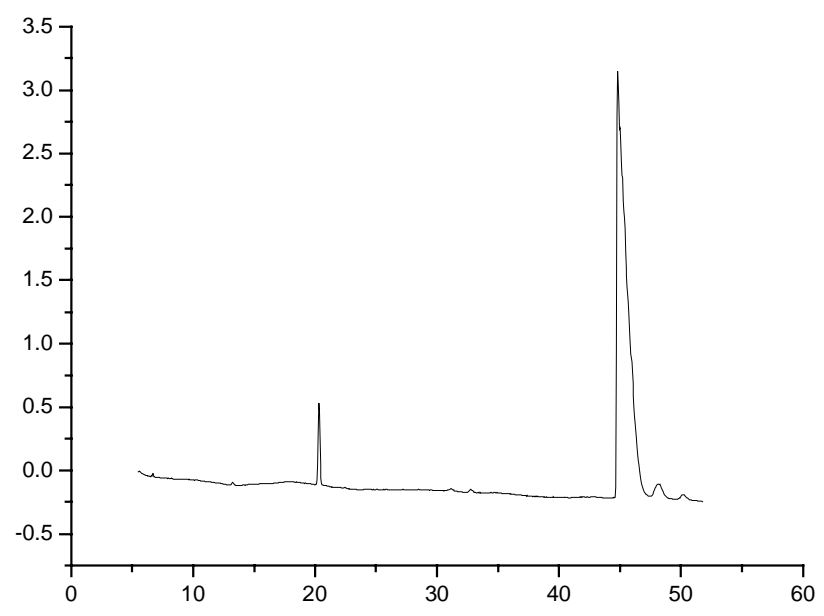

\section{Reference}

(1) Levison, J. J.; Robinson, S. D. J. Chem. Soc. (A), 1970, 2947.

(2) Evans, D, ; Yagupsky, G.; Wilkinson, G. J. Chem. SoC. (A), 1968, 2660. 